\title{
Broadband boundary effects on Brownian motion
}

\author{
Jianyong Mo, Akarsh Simha, and Mark G. Raizen* \\ Center for Nonlinear Dynamics and Department of Physics, The University of Texas at Austin, Austin, Texas 78712, USA
}

(Received 2 September 2015; published 2 December 2015)

\begin{abstract}
Brownian motion of particles in confined fluids is important for many applications, yet the effects of the boundary over a wide range of time scales are still not well understood. We report high-bandwidth, comprehensive measurements of Brownian motion of an optically trapped micrometer-sized silica sphere in water near an approximately flat wall. At short distances we observe anisotropic Brownian motion with respect to the wall. We find that surface confinement not only occurs in the long time scale diffusive regime but also in the short time scale ballistic regime, and the velocity autocorrelation function of the Brownian particle decays faster than that of a particle in bulk fluid. Furthermore, at low frequencies the thermal force loses its color due to the reflected flow from the no-slip boundary. The power spectrum of the thermal force on the particle near a no-slip boundary becomes flat at low frequencies. This detailed understanding of boundary effects on Brownian motion opens a door to developing a 3D microscope using particles as remote sensors.
\end{abstract}

DOI: 10.1103/PhysRevE.92.062106

\section{INTRODUCTION}

Understanding the influence of boundaries on particle dynamics is of great significance, from both a fundamental and an applied point of view [1-9]. For example, blood cells moving in vessels, particles migrating in porous media, and macromolecules diffusing in membranes are all affected by the presence of boundaries. It is well known that the mobility of particles decreases as they approach boundaries at which the fluid does not slip. This effect of "surface confinement" was predicted by Lorentz in 1907 [10]. The increase of the drag force is attributed to the alteration of the hydrodynamic interaction between the particle and the fluid generated by the no-slip boundaries. The motion of the particle becomes anisotropic because the drag force parallel to the wall is typically less than that perpendicular to the wall. This effect is significant when the dimensions of the confining geometry and the suspended particles are comparable. There have been many experiments verifying this surface confinement effect, mainly focusing on measuring the changes in the diffusion coefficient of micron-sized particles near surfaces using dynamic light scattering [11], video microscopy [12], total internal reflection microscopy [13], or oscillating optical tweezers [14]. However, all these experiments have only addressed boundary effects in the long time scale diffusive regime of Brownian motion.

In addition to surface confinement, it has been reported that the presence of a no-slip boundary also affects the velocity autocorrelation function (VACF) of a Brownian particle $[15,16]$. The long time tail of the VACF in the bulk case is largely canceled by reflected flow from the wall, resulting in a more quickly decaying VACF. Boundary effects on the position power spectral density (PSD) in a limited frequency window have also been reported [17,18]. These experiments have observed a resonance caused by the color of the thermal force, and its suppression as the sphere-wall separation decreases. A broadband, comprehensive experimental study of effects of the boundary via hydrodynamic interaction on Brownian motion is still lacking in spite of the previous work on boundary effects

\footnotetext{
*raizen@physics.utexas.edu
}

PACS number(s): 05.40.Jc, 47.61.-k, 87.15.Vv, 87.80.Cc

mentioned above [11-18] and the fact that Brownian motion of a sphere in bulk has been well studied [18-22].

Here we investigate the effects of a boundary on the Brownian motion of a sphere in water both in the diffusive regime and the ballistic regime. It is important to note that the boundary effects in this article occur only through hydrodynamic interaction. Other close-range forces between particles and surface, like the electrostatic force [23], van der Waals force [24], and Casimir force [25] can be neglected because we keep the sphere-wall separation $(\geqslant 3 \mu \mathrm{m})$ much larger than the length scales at which those forces need to be considered. The point approximation [26] used in presently available theoretical models for Brownian motion with boundary effects also sets a lower bound on the sphere-wall separation of interest.

This paper is organized as follows. In Sec. II, we review the theory of Brownian motion near a flat wall with no-slip boundary conditions. In Sec. III, we describe our experimental setup. In Sec. IV, we describe our calibrations and data analysis procedures. In Sec. V, we present and discuss our experimental results. In Sec. VI, we discuss a numerical simulation performed using COMSOL Multiphysics software. We conclude with a discussion in Sec. VII.

\section{THEORY}

\section{A. Statistical properties of Brownian motion}

The position $\mathbf{x}(t)$ and velocity $\mathbf{v}(t)=\dot{\mathbf{x}}(t)$ of a Brownian particle in equilibrium may be regarded as stationary and ergodic random processes. Our experimental study of Brownian motion involves the description of various statistical properties that we shall define below. The parentheses 〈〉 denote equilibrium ensemble averages, which may be reinterpreted as time averages through the assumption of ergodicity.

To a good approximation, the dynamics of the three spatial dimensions are decoupled from each other. Hence we shall usually consider one spatial component of the position vector $\mathbf{x}(t)$, and denote it by $x(t)$. The corresponding velocity component will be denoted as $v(t)=\dot{x}(t)$. The position autocorrelation function (PACF) of the particle is defined through $\operatorname{PACF}(t) \equiv$ $\langle x(t+\tau) x(\tau)\rangle$. The mean-square displacement (MSD) of the particle is defined as $\operatorname{MSD}(t) \equiv\left\langle[x(t+\tau)-x(\tau)]^{2}\right\rangle$. 
The velocity autocorrelation function (VACF) of the particle is defined through $\operatorname{VACF}(t) \equiv\langle v(t+\tau) v(\tau)\rangle$. The position power spectral density (PSD) and velocity power spectral density (VPSD) are, by the Wiener-Khinchin theorem, the Fourier transforms of the PACF and VACF, respectively.

In the hydrodynamic theory of Brownian motion, the Green-Kubo formula is used to relate these statistical properties to the admittance of the particle coupled to the fluid, which is the linear velocity response of the particle to an applied external force, whose theoretical computation is discussed in the following subsection.

\section{B. Computation of the admittance}

Here, we review briefly a modified form of the theoretical work of Felderhof [26] on the Brownian motion of a spherical particle in an incompressible fluid near a flat and infinite noslip wall. In this work, we shall denote the radius of the particle by $a$, and the distance to the wall as measured from the center of the sphere by $h$. The modifications to Felderhof's theory will be justified and discussed in detail in a future publication [27].

Felderhof's framework assumes that the sphere may be approximated by a point force for purposes of calculating the fluid flow. The small amplitude incompressible fluid motion is governed by the linearized incompressible Navier-Stokes equations,

$$
\rho_{f} \frac{\partial \mathbf{v}}{\partial t}=\eta \Delta \mathbf{v}-\nabla P, \quad \nabla \cdot \mathbf{v}=0,
$$

where $\mathbf{v}$ and $P$ denote the velocity and pressure fields respectively, $\eta$ is the dynamic shear viscosity of the fluid, and $\rho_{f}$ is the density of the fluid. Upon Fourier analysis in time, the first equation transforms into

$$
\eta\left(\Delta \mathbf{v}_{\omega}-\alpha^{2} \mathbf{v}_{\omega}\right)-\nabla P_{\omega}=0,
$$

where $\mathbf{v}_{\omega}, P_{\omega}$ denote the Fourier transforms of $v$ and $P$, respectively, and we have used the notation $\alpha=$ $\left(-i \omega \rho_{f} / \eta\right)^{1 / 2}, \operatorname{Re}[\alpha]>0$ for the complex wave number that appears in the equation. We remark that by taking the curl of the above equation, it is seen that the vorticity, defined as the $\nabla \times \mathbf{v}$, satisfies a Helmholtz equation with this wave number.

The equation of motion for the particle can be written as

$$
-i \omega\left(m_{p}-m_{f}\right) \mathbf{U}_{\omega}=\mathbf{E}_{\omega}-\mathcal{F}_{\omega},
$$

where $\mathbf{U}_{\omega}$ is the particle velocity, $\mathbf{E}_{\omega}$ is an external force applied on the particle, and $\mathcal{F}_{\omega}$ is the induced force [28]; $m_{p}$ and $m_{f}$ are the masses of the particle and the displaced fluid, respectively. Within the point particle approximation, inhomogeneity of the flow over the scale of the particle is ignored and the induced force is calculated using the generalized Faxén theorem [29] with the incident flow field replaced by the flow at the center of the sphere. The proposed expression for $\mathcal{F}_{\omega}$ is given by [27]

$$
\mathcal{F}_{\omega}=\left[\gamma_{s}(1+\alpha a)-\frac{3}{2} i \omega m_{f}\right]\left[\mathbf{U}_{\omega}-\mathrm{R} \cdot \mathcal{F}_{\omega}\right],
$$

where $\gamma_{s}=6 \pi \eta a$ is the steady Stokes friction coefficient, and $R$ is the reaction field tensor as defined and calculated by Felderhof [26]. The reaction field tensor, when contracted with the point force that represents the presence of the particle, yields the velocity of the reflected flow from the wall at the position of the sphere, which enters the generalized Faxén theorem in the point particle approximation. In the above, Felderhof's expression for $\mathcal{F}_{\omega}$ has been modified to use the point force that correctly reproduces the far-field behavior of a sphere [27]. The equation may be rearranged appropriately to solve for $\mathcal{F}_{\omega}$ in terms of the particle velocity $\mathbf{U}_{\omega}$.

Three time scales occur in the description of Brownian motion near a plane wall $[16,18]: \tau_{f}=a^{2} \rho_{f} / \eta$, the time scale over which the vorticity diffuses over the size of the spherical particle; $\tau_{w}=h^{2} \rho_{f} / \eta$, the time scale over which the vorticity diffuses the sphere-wall distance; and $\tau_{p}=2 a^{2} \rho_{p} /(9 \eta)$, the momentum relaxation time of the microsphere. Here, $\rho_{p}$ denotes the density of the spherical particle.

The admittance tensor $\mathcal{Y}(\omega)$, defined through $\mathbf{U}_{\omega}=\mathcal{Y}(\omega)$. $\mathbf{E}_{\omega}$, is a useful quantity for the characterization of the linear response of the particle's velocity to thermal fluctuations [26]. It may be computed using Eqs. (3) and (4). Once the admittance tensor is known, various statistical properties of Brownian motion may be computed.

By application of the Green-Kubo formula, the velocity autocorrelation function $\mathrm{C}_{v}(t)$ is related to the frequencydependent admittance [26] through

$$
\mathcal{Y}(\omega)=\frac{1}{k_{B} T} \int_{0}^{\infty} d t e^{i \omega t} \mathrm{C}_{v}(t)
$$

where $k_{B}$ is Boltzmann's constant and $T$ is the absolute temperature. It must be noted that the short-time behavior of $\mathrm{C}_{v}(t)$ obtained from Felderhof's theory is in disagreement with the modified equipartition theorem [30] using well-known results for the effective mass of a sphere in a bounded fluid [31]. The proposed correction [27] to Felderhof's theory resolves this issue.

The position and velocity power spectral densities can also be calculated from the admittance using [16]

$$
\mathrm{S}_{x}(\omega)=\frac{2 k_{B} T \operatorname{Re}[\mathcal{Y}(\omega)]}{\omega^{2}}
$$

and

$$
\mathrm{S}_{v}(\omega)=2 k_{B} T \operatorname{Re}[\mathcal{Y}(\omega)]
$$

For a spherical Brownian particle trapped in a harmonic potential in an unbounded fluid, the (scalar) admittance is given by

$$
\mathcal{Y}_{0}(\omega)=\frac{-i \omega}{-m \omega^{2}-i \omega \gamma(\omega)+K},
$$

where $K$ is the trap stiffness constant and $\gamma(\omega)=\gamma_{s}(1+$ $\left.\sqrt{-i \omega \tau_{f}}-i \omega \tau_{f} / 9\right)$. The proposed expression for the admittance in the presence of the boundary, but without a harmonic trap is given by [27]

$$
\mathcal{Y}_{B}(\omega)=\frac{\left[1+\gamma_{s}\left(1+\sqrt{-i \omega \tau_{f}}-i \omega \tau_{f} / 3\right) \mathrm{R}(\omega)\right]}{i \omega\left(m_{f}-m_{p}\right)\left[\gamma_{s}\left(1+\sqrt{-i \omega \tau_{f}}-i \omega \tau_{f} / 3\right) \mathrm{R}(\omega)\right]+\left(\gamma(\omega)-i \omega m_{p}\right)} .
$$


The tensors $\mathcal{Y}$ and $\mathrm{R}$ in our case are diagonal [26], whereby no ambiguity is caused by their occurrence in the denominators of equations. We shall denote the relevant components of $\mathrm{R}$ by $R_{\|}$[corresponding to Eq. (3.9) in Ref. [26]] and $R_{\perp}$ [corresponding to Eq. (3.10) in Ref. [26]; also see erratum [32]]. The admittance in the presence of the boundary, including the effect of a harmonic trap is then given by [16]

$$
\mathcal{Y}_{B}^{K}(\omega)=\frac{-i \omega \mathcal{Y}_{B}(\omega)}{-i \omega+K \mathcal{Y}_{B}(\omega)}
$$

With this admittance, one can calculate all the statistical properties of a trapped Brownian particle near a wall using Eqs. (5)-(7) and the procedures described in Sec. IV.

The drag coefficients in the presence of the boundary are related to the admittance and reaction field tensor through

$$
\begin{aligned}
\gamma_{B}(\omega) & =\frac{1}{\mathcal{Y}_{B}^{K}(\omega)}+i \omega m_{p}+\frac{K}{i \omega} \\
& =\frac{1}{\mathcal{Y}_{B}(\omega)}+i \omega m_{p} \\
& =\frac{\gamma(\omega)+\gamma_{s}\left(1+\sqrt{-i \omega \tau_{f}}-i \omega \tau_{f} / 3\right)\left(i \omega m_{f}\right) \mathrm{R}(\omega)}{\left[1+\gamma_{s}\left(1+\sqrt{-i \omega \tau_{f}}-i \omega \tau_{f} / 3\right) \mathrm{R}(\omega)\right]} .
\end{aligned}
$$

In an unbounded fluid, the effective inertial mass of a spherical particle is given by the mass of the particle $m_{p}$, plus an added mass of $m_{a}=m_{f} / 2$, where $m_{f}$ is the mass of the liquid displaced by the particle [30]. The presence of the boundary alters this effective mass. The effective mass becomes anisotropic and also depends on the distance to the wall. Near a flat wall, the expressions for the effective mass in the perpendicular and parallel directions are given by

$$
\begin{aligned}
& m_{\perp}^{*}=m_{p}+\frac{m_{f}}{2}\left[1+\frac{3}{8}\left(\frac{a}{h}\right)^{3}\right], \\
& m_{\|}^{*}=m_{p}+\frac{m_{f}}{2}\left[1+\frac{3}{16}\left(\frac{a}{h}\right)^{3}\right],
\end{aligned}
$$

respectively [31]. The modified energy equipartition theorem prediction [30] for the mean-squared thermal velocity of the hydrodynamically coupled particle is thus

$$
\left\langle\left(v^{*}\right)^{2}\right\rangle=k_{B} T / m^{*},
$$

where $m^{*}$ is either $m_{\perp}^{*}$ or $m_{\|}^{*}$ for velocities perpendicular or parallel to the boundary respectively.

\section{Statistical properties of the thermal force}

Brownian motion with memorylike damping can also be described and solved using a generalized Langevin equation (GLE) [33] in which the damping is expressed as convolution of the velocity $\dot{\mathbf{x}}(t)$ with a memory kernel. For a Brownian particle in a harmonic potential, the GLE is most often written as

$$
m_{p} \ddot{\mathbf{x}}(t)+\int_{-\infty}^{t} \zeta\left(t-t^{\prime}\right) \dot{\mathbf{x}}\left(t^{\prime}\right) d t^{\prime}+K \mathbf{x}(t)=\mathbf{F}_{\mathrm{th}}(t),
$$

where $\mathbf{x}(t)$ denotes the position of the Brownian particle, and $\zeta\left(t-t^{\prime}\right)$ is a memory kernel tensor that comes from the drag exerted on the particle by the fluid. The thermal force $\mathbf{F}_{\text {th }}(t)$ is a random process that captures the effect of thermal fluctuations in the fluid on the particle. In the frequency domain, we may write the GLE as

$$
\left[-m_{p} \omega^{2}-i \omega \gamma_{B}(\omega)+K\right] \widehat{\mathbf{x}}(\omega)=\widehat{\mathbf{F}}_{\mathrm{th}}(\omega),
$$

where the hats indicate Fourier transformed quantities. The statistics of the thermal force are related to the drag coefficient $\gamma_{B}$ through the fluctuation-dissipation theorem [34].

The one-sided thermal force power spectral density $\mathrm{S}_{F}(\omega)$ is related to the drag coefficient $\gamma_{B}(\omega)$ through a form of the fluctuation-dissipation theorem [18,34],

$$
\mathrm{S}_{F}(\omega)=4 k_{B} T \operatorname{Re}\left[\gamma_{B}(\omega)\right] .
$$

In the Einstein-Ornstein-Uhlenbeck model [35] of Brownian motion, which is valid only when the inertia of the fluid is negligible, the corresponding thermal force is white noise, with the flat (one-sided) spectrum $S_{F}=4 k_{B} T \gamma_{s}$. Addition of the Basset force [33] to the Einstein-Ornstein-Uhlenbeck model to account for the history of the fluid flow induced by the particle's motion results in the colored thermal force spectral density $S_{F}=4 k_{B} T \gamma_{s}\left(1+\sqrt{\omega \tau_{f} / 2}\right)$ on a particle in an unbounded fluid. Surprisingly, the thermal force loses its color at low frequencies in the presence of a boundary. The thermal force power spectral density (FPSD) on the particle near a no-slip wall becomes flat at low frequencies (when $\omega \tau_{f} \ll 1$ and $\left.\omega \tau_{w} \ll 1\right)$.

The low frequency asymptotic behavior of the thermal force PSD on a particle near a flat wall is found by expanding the reaction field tensor and the expression for the drag coefficient to lowest few orders in $\omega$, and is valid for $\omega \ll 1 / \tau_{f}, 1 / \tau_{w}$. We find that, as $\omega \rightarrow 0$, the asymptotic forms of the (one-sided) PSD of the thermal force on the particle in the parallel and perpendicular directions near a no-slip flat wall are given by

$$
\begin{aligned}
S_{F, \|}(\omega) & =4 k_{B} T \operatorname{Re}\left[\gamma_{\|}(\omega)\right] \\
& \sim 4 k_{B} T \gamma_{s}\left(\frac{1}{1-\frac{9 a}{16 h}}+\frac{128 \sqrt{2}\left(3 h^{4}-a^{2} h^{2}\right)}{3 a^{2}(16 h-9 a)^{2}}\left(\omega \tau_{f}\right)^{\frac{3}{2}}\right), \\
S_{F, \perp}(\omega) & =4 k_{B} T \operatorname{Re}\left[\gamma_{\perp}(\omega)\right] \\
& \sim 4 k_{B} T \gamma_{s}\left(\frac{1}{1-\frac{9 a}{8 h}}-\frac{32 \sqrt{2} h^{2}}{3(9 a-8 h)^{2}}\left(\omega \tau_{f}\right)^{\frac{3}{2}}\right),
\end{aligned}
$$

respectively. In contrast, the (one-sided) PSD of the thermal force on the sphere in bulk is given by

$$
S_{F}(\omega)=4 k_{B} T \operatorname{Re}[\gamma(\omega)]=4 k_{B} T \gamma_{s}\left(1+\sqrt{\frac{\omega \tau_{f}}{2}}\right) .
$$

The enhanced flatness of the thermal force PSD observed in the presence of a no-slip plane wall, which can be understood as resulting from destructive interference of the reflected flow, is seen in the above expressions through the lack of $\sqrt{\omega \tau_{f}}$ and $\omega \tau_{f}$ terms. In contrast, this effect is not seen in the parallel direction in the case of a perfect slip plane wall, presumably due to constructive interference from the reflected flow. However, in the perpendicular direction to a perfect slip wall, the flatness is still seen, presumably due to the no penetration boundary condition keeping the reflected flow out of phase. 
For completeness, we give the results for a perfect slip flat boundary as well [36]. The asymptotic forms of the (one-sided) PSD of the thermal force in the parallel and perpendicular directions, on a sphere near a perfect-slip boundary for $\omega \tau_{f}, \omega \tau_{w} \ll 1$, are given by

$$
\begin{aligned}
& S_{F, \|}^{\mathrm{slip}}(\omega) \sim 4 k_{B} T \gamma_{s}\left(\frac{1}{1+\frac{3 a}{8 h}}+\frac{64 \sqrt{2} h^{2}}{(3 a+8 h)^{2}}\left(\omega \tau_{f}\right)^{\frac{1}{2}}\right), \\
& S_{F, \perp}^{\operatorname{slip}}(\omega) \sim 4 k_{B} T \gamma_{s}\left(\frac{1}{1-\frac{3 a}{4 h}}+\frac{8 \sqrt{2}\left(6 h^{4}-5 a^{2} h^{2}\right)}{15 a^{2}(4 h-3 a)^{2}}\left(\omega \tau_{f}\right)^{\frac{3}{2}}\right),
\end{aligned}
$$

respectively.

\section{EXPERIMENT}

A simplified schematic of our experimental setup for measuring the Brownian motion of a micrometer-sized silica sphere in water near an approximately flat wall, both in perpendicular and parallel directions, is shown in Fig. 1. An approximately $3 \mu \mathrm{m}$ diameter silica glass microsphere is trapped by a $1064 \mathrm{~nm}$ laser (Mephisto, Innolight) focused by a water-immersion microscope objective (LOMO OM-25, numerical aperture 1.23). The laser is introduced into the objective through a polarization-maintaining single-mode fiber (Thorlabs, P3-1064PM-FC-5) followed by a $200 \mathrm{~mm}$ tube lens. The trapping laser beam also serves as the detection beam, which is collected by an identical objective and then split into two roughly equal halves in the horizontal direction in the laboratory frame using a D-shaped mirror (Thorlabs, BBD05-E03). An AC-coupled, homemade, high-bandwidth balanced detector [21] is used to amplify the power difference between the halves, which depends linearly on the position of

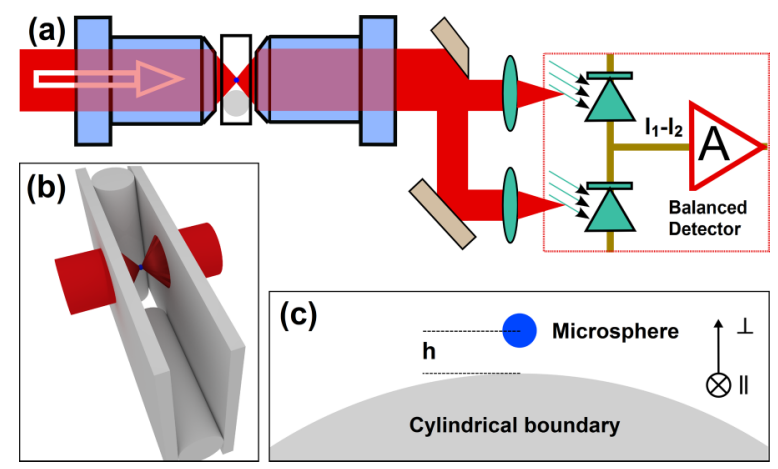

FIG. 1. (Color online) Schematic of setup to study the Brownian motion of an optically trapped glass microsphere in water near a boundary. (a) Simplified schematic showing a glass microsphere (3 $\mu \mathrm{m}$ diameter) trapped at the focus of a $1064 \mathrm{~nm}$ optical tweezer near a boundary. The trapping beam is used to detect the horizontal motion of the particle using a high bandwidth balanced detection system [21]. (b) Two $80 \pm 1 \mu \mathrm{m}$ diameter cylindrical fibers are sealed in the chamber with their axes in the vertical and horizontal directions, respectively, providing well-defined no-slip cylindrical boundaries. (c) The geometry of the sphere and cylindrical boundary to scale. The sphere-wall separation $h$ refers to the distance between the center of the sphere and the surface of the boundary. The perpendicular $(\perp)$ and parallel $(\|)$ directions referred to in the text are as marked. the trapped particle. These aspects of the setup are shown in a simplified manner in Fig. 1(a). The position of the D-shaped mirror is adjusted before every data acquisition to balance the laser power in the two halves. We also set up optics to allow redirection of the detection beam to a DC-coupled balanced detector (Thorlabs PDB120C) to enable calibration procedures described in Sec. IV B. In this experiment, the trapping laser beam power is around $400 \mathrm{~mW}$ and the detection beam power is set to about $150 \mathrm{~mW}$.

The fluid chamber is constructed within a layer of nescofilm (Bando Chemical Ind. LTD., $\sim 80 \mu \mathrm{m}$ thickness) sandwiched between two number 0 microscope coverslips (Ted Pella, $\sim 100 \mu \mathrm{m}$ thickness). Two $80 \pm 1 \mu \mathrm{m}$ diameter cylindrical glass fibers (Thorlabs SM980G80, with coating layer stripped) are heat sealed in the chamber with their axes in the vertical and horizontal directions, respectively, providing two well-defined cylindrical boundaries with no-slip boundary conditions in either direction. This is done so as to enable measurement of the Brownian motion in perpendicular and parallel directions to the wall independently with vertical and horizontal fibers, respectively, since our detection system measures the horizontal motion of the microsphere. A 3D schematic of this is shown in Fig. 1(b). The geometry of the sphere and cylindrical boundary is shown to scale in Fig. 1(c). The cylindrical boundary can be approximated as a flat wall when the sphere-wall separation $h$ and the size of the sphere $a$ are much smaller than the radius of the fiber, as shown later in the text.

The cylindrical boundary is intentionally chosen to avoid clipping the laser beam with the boundary for small spherewall separations. In our setup, the waist of the trapping beam is estimated to be around $1 \mu \mathrm{m}$, and as long as the spherewall separation $h$ is more than $3 \mu \mathrm{m}$, the clipping effect can be neglected. We confirm the lack of clipping by monitoring the trapping beam transmission through the chamber and by the observed stability of the fitting parameters at different positions.

The position of the chamber is adjusted so that the optical trap confines the particle to the center of the chamber in the beam propagation direction. This is to avoid coverslip boundary effects, which may then be neglected since the sphere-coverslip separation is $\sim 40 \mu \mathrm{m}$, which is considerably larger than the size of the sphere.

The whole chamber is mounted onto a piezo stage (two Thorlabs DRV517, with controller BPC301 and a strain gauge giving relative positions), which gives us the ability to position the cylindrical fibers with a precision of $\sim 10 \mathrm{~nm}$ in two directions, in order to control the sphere-wall separation. There are two main sources of fluctuations in the sphere-wall separation: relative vibration motion between the fibers and laser trapping focus, and the thermal motion of the microsphere in the trap. The relative vibration is reduced to on the order of $10 \mathrm{~nm}$ by using a gas floating optical table (TMC Vibration Control). The thermal motion fluctuations in the trap can be reduced by increasing the trap strength, resulting $\sqrt{k_{B} T / K} \sim 10 \mathrm{~nm}$ in our system. The piezo stage PID control loop is kept open while taking data to avoid additional noise introduced by it. In Sec. V, the boundary effects on the motion of a sphere at four positions, with sphere-wall separations of $30 \mu \mathrm{m}, 6.1 \mu \mathrm{m}, 4.6 \mu \mathrm{m}$, and $3.1 \mu \mathrm{m}$, will be presented and discussed in detail. 

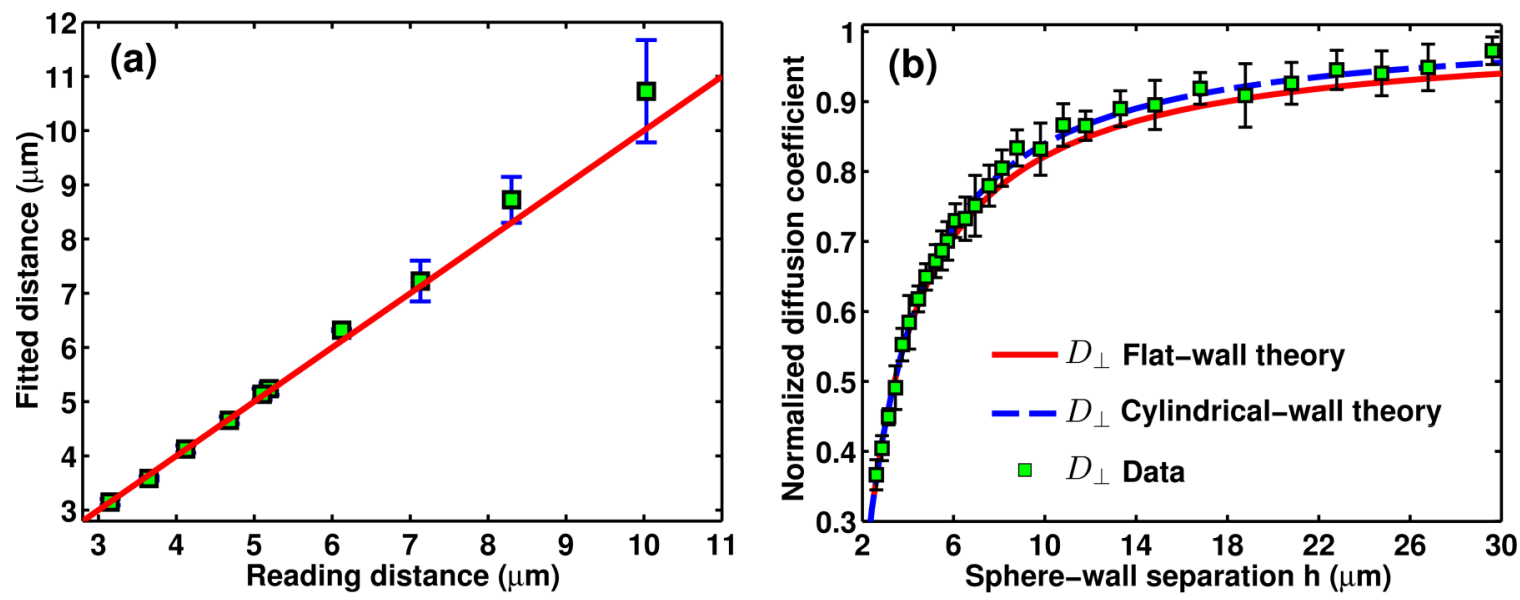

FIG. 2. (Color online) Measurements of the absolute sphere-wall separations in the perpendicular direction with a $3 \mu \mathrm{m}$ diameter silica microsphere. (a) Obtained from fitting the data recorded by the AC-coupled detector to the no-slip flat-wall theoretical VACF. The green squares with error bars represent the experimental data and statistical errors obtained by averaging from 10 measurements at each position. The horizontal axis denotes the distance measured by the strain gauge after subtraction of the fitted offset (discussed in the text). The red line is the $y=x$ line; the VACF fitting gives reliable results as long as the sphere-wall separation is smaller than $7 \mu \mathrm{m}$. (b) Obtained from measuring the hindered diffusion coefficient using the DC-coupled detector with sphere-wall separations from $2.5 \mu \mathrm{m}$ to $30 \mu \mathrm{m}$. The red line is the flat-wall theory, and the blue dashed line is the modified cylindrical-wall theory (see text). The green squares with error bars represent the experimental data and statistical errors obtained by averaging from 10 measurements at each position.

Experiments are performed using silica microspheres (Bangs Laboratories SS05N, $n=1.46, \rho_{p}=2.0 \mathrm{~g} / \mathrm{cm}^{3}$ ) in HPLC-grade water $\left(n=1.33, \rho_{f}=1 \mathrm{~g} / \mathrm{cm}^{3}, \eta=10^{-3} \mathrm{~Pa} \mathrm{~s}\right)$ at $22 \pm 1{ }^{\circ} \mathrm{C}$. High sphericity of the microspheres is necessary to eliminate the rotational motion contribution due to asymmetry of the microspheres, and is confirmed by scanning electron microscope images [22].

The voltage signal from the balanced detector is recorded by a 16-bit digitizer (GaGe applied, CS1622) at a sampling rate of $200 \mathrm{MSa} / \mathrm{s}$. Each continuous trajectory contains $2^{27}$ samples, corresponding to about $0.7 \mathrm{~s}$ acquisition time. We take 10 such measurements at each position. The voltage signal from the digitizer is, to a very good approximation, proportional to the displacement of the particle in the trap. This calibration factor is determined by fitting of the measurements to theory as described in Sec. IV A.

\section{CALIBRATION AND DATA ANALYSIS}

\section{A. Calibration of the tweezers and computation of statistical properties}

In this section, we describe the calibration methods and the data analysis techniques used to obtain the results presented in Sec. V. The numerical computation, of both theoretical predictions and measured values of the various statistical properties characterizing Brownian motion that were defined in Sec. II A, is outlined.

We first compute the voltage power spectral density (voltage PSD) by means of a fast Fourier transform (FFT) of the voltage signal, followed by taking the magnitude squared and appropriately scaling it [37]. The measured magnitude of the transfer function of the detection system is then squared and divided out of this PSD [21].

The Wiener-Khinchin theorem states that for wide-sense stationary random processes, the power spectral density and autocorrelation are Fourier transform pairs. We make use of this to calculate the PACF, up to a calibration constant, by FFT of the voltage PSD. In doing so, we discard data points at frequencies much lower than the trap frequency $F_{k}=1 /\left(2 \pi \tau_{k}\right)$, which are plagued by low-frequency noise in the system, and assume that the power spectrum is flat in this region. We then calculate the experimental MSD, up to a calibration constant, from the PACF. In a similar manner, we numerically compute the theoretical MSD from the theoretical PACF, by a Filon Fourier transform [38] of the analytic expression for the position PSD given by Eq. (6).

A least-squares fit of the MSD of the recorded trajectories to the theory of Brownian motion near a no-slip flat wall $[16,26,32]$ is used to determine the sphere diameter $d=$ $2 a$, the trap stiffness $K$, and the volts-per-meter calibration factor $C$. The fitting results for the sphere diameter $(d=$ $3.06 \pm 0.19 \mu \mathrm{m})$, trap stiffness $(K=151 \pm 31 \mu \mathrm{N} / \mathrm{m})$, and volts-per-meter calibration factor $(C=20.1 \pm 2.0 \mathrm{mV} / \mathrm{nm})$ are similar at those four positions (with sphere-wall separations of $30 \mu \mathrm{m}, 6.1 \mu \mathrm{m}, 4.6 \mu \mathrm{m}$, and $3.1 \mu \mathrm{m}$ ), which indicates that the laser is not clipped by the cylindrical boundary. The uncertainty of each fit parameter is determined from the variance in the results of independent MSD fits of 10 trajectories per position.

Once the calibration factor $C$ is determined, the voltage signal may be calibrated to a position signal. The velocity signal is then computed by means of a discrete derivative operator applied to the position signal. The VPSD may be then calculated from the velocity signal in a similar manner to the position PSD. The VACF of the measured trajectory is then obtained through FFT of the VPSD. The theoretical VPSD is similarly computed through the analytic expression (7), and the theoretical prediction for the VACF is numerically computed from the VPSD using the Filon Fourier transform algorithm.

The piezo stage only gives us the relative distances between different positions of the particle. As described in detail in 

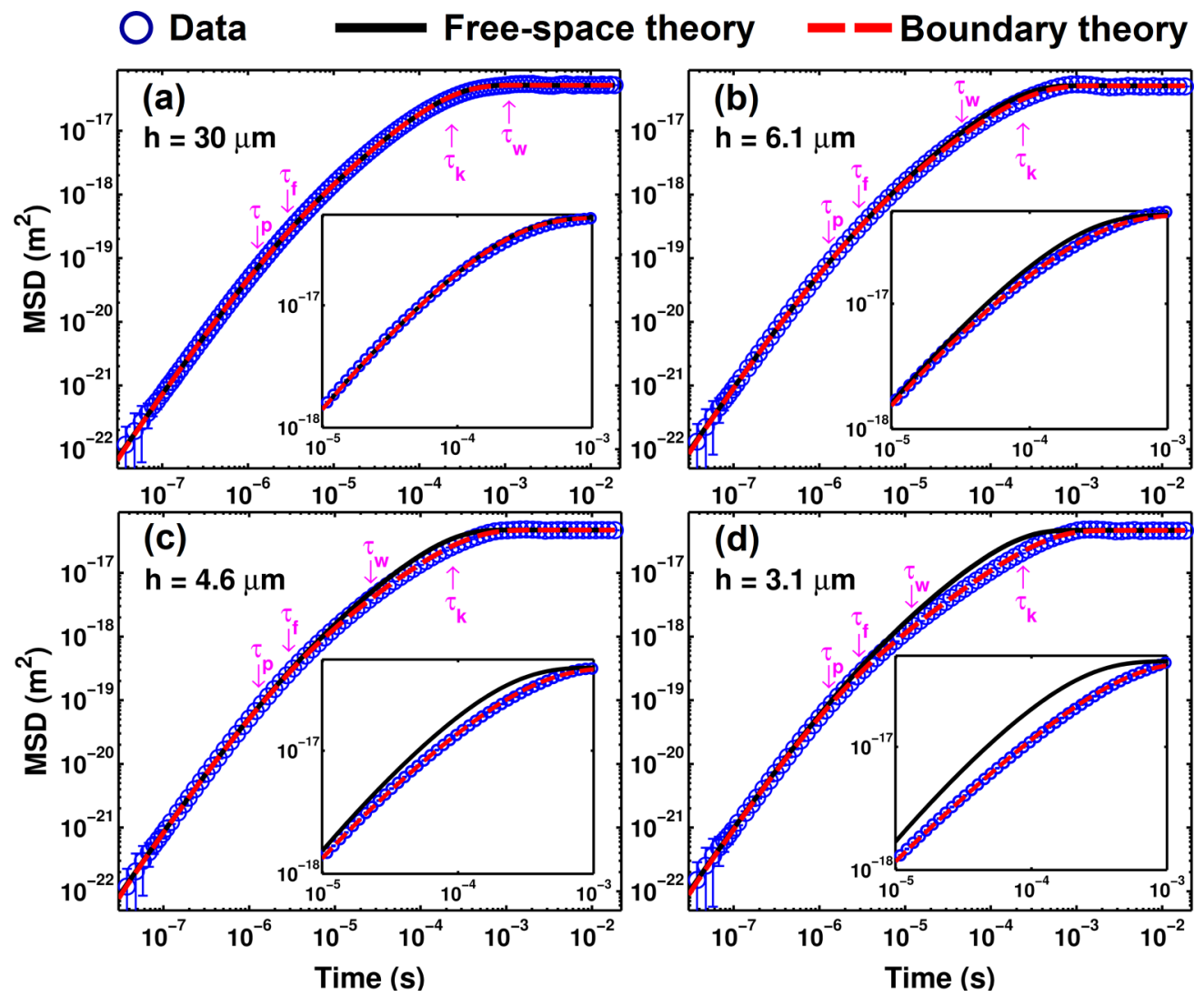

FIG. 3. (Color online) Experimental and theoretical mean square displacements (MSDs) at four positions in the perpendicular direction with respect to the wall. (a) With a sphere-wall separation ( $h$ ) of $30 \mu \mathrm{m}$ ( $\tau_{w}=0.9 \mathrm{~ms}$ ). (b) $h=6.1 \mu \mathrm{m}\left(\tau_{w}=37 \mu \mathrm{s}\right)$. (c) $h=4.6 \mu \mathrm{m}$ $\left(\tau_{w}=21 \mu \mathrm{s}\right)$. (d) $h=3.1 \mu \mathrm{m}\left(\tau_{w}=9.6 \mu \mathrm{s}\right)$. The blue circles are the experimental data $\left(\tau_{p}=1 \mu \mathrm{s}, \tau_{f}=2.3 \mu \mathrm{s}, \tau_{k}=190 \mu \mathrm{s}\right)$; the black lines are the unbounded theoretical predictions [33] and the red dashed lines correspond to the bounded theoretical predictions at various sphere-wall separations. The MSD becomes suppressed as the sphere approaches the wall. The insets show higher resolution of the suppression of the MSD.

Sec. IV B, the sphere-wall separation $h$ may be determined by fitting of the VACF to theoretical predictions.

For each acquisition of Brownian motion with a sphere, we also make an acquisition of noise from the detection laser with no sphere in the trap. For spectra and correlation functions, we analyze the noise acquisition in the same way as the data and subtract it to remove the contribution of noise from that of the sphere's motion. This method is valid as long as the noise is not correlated to the sphere's motion. The error bars in Figs. 3-8 represent two sources of error: the first is statistical error, calculated from distributions of points within each "block" used for plotting data [37]. The second is the uncertainty in the amplitude of the subtracted noise, which is significant at high frequencies (or short time scales), where noise dominates the signal.

The measured stochastic thermal force power spectral density (FPSD) is numerically calculated from the measured position PSD by inverting the generalized Langevin equation in the frequency domain [Eq. (15)]. The Green's function for the GLE may thus be written as [18]

$$
\widehat{\mathrm{G}}(\omega)=\left[-\omega^{2} m_{p}-i \omega \gamma_{B}(\omega)+K\right]^{-1},
$$

and is used to compute the FPSD by evaluating

$$
\mathrm{S}_{F}(\omega)=|\widehat{\mathrm{G}}(\omega)|^{-2} \mathrm{~S}_{x}(\omega)
$$

numerically. We remark that this procedure assumes the hydrodynamic theory for the drag coefficient, but does not assume the validity of the fluctuation-dissipation theorem.

The theoretical FPSD is computed from the analytic expression for the drag coefficient [Eq. (11)] and the fluctuationdissipation theorem [Eq. (16)].

The observation that the thermal force PSD calculated from experimental data matches the theoretical prediction within experimental error (see Sec. V) can be interpreted as a verification of the fluctuation-dissipation theorem subject to the assumption that the hydrodynamic theory is correct.

\section{B. Calibration of the absolute sphere-wall separation}

The piezo stage used in the experiment gives us precise measurements of the position of the particle relative to an arbitrary origin. It is not possible to determine the absolute distance to the wall precisely from the optical image of the chamber due to diffraction fringes around and errors in adjusting the focal plane. The absolute separations between the sphere and the wall $(h)$ reported in this paper are obtained by calibrating the piezo against results from least-squares fitting of the experimental VACF to the theoretical predictions from the no-slip flat wall boundary theory $[16,26,32]$. In principle, any of the statistical properties can be fit to the theoretical 


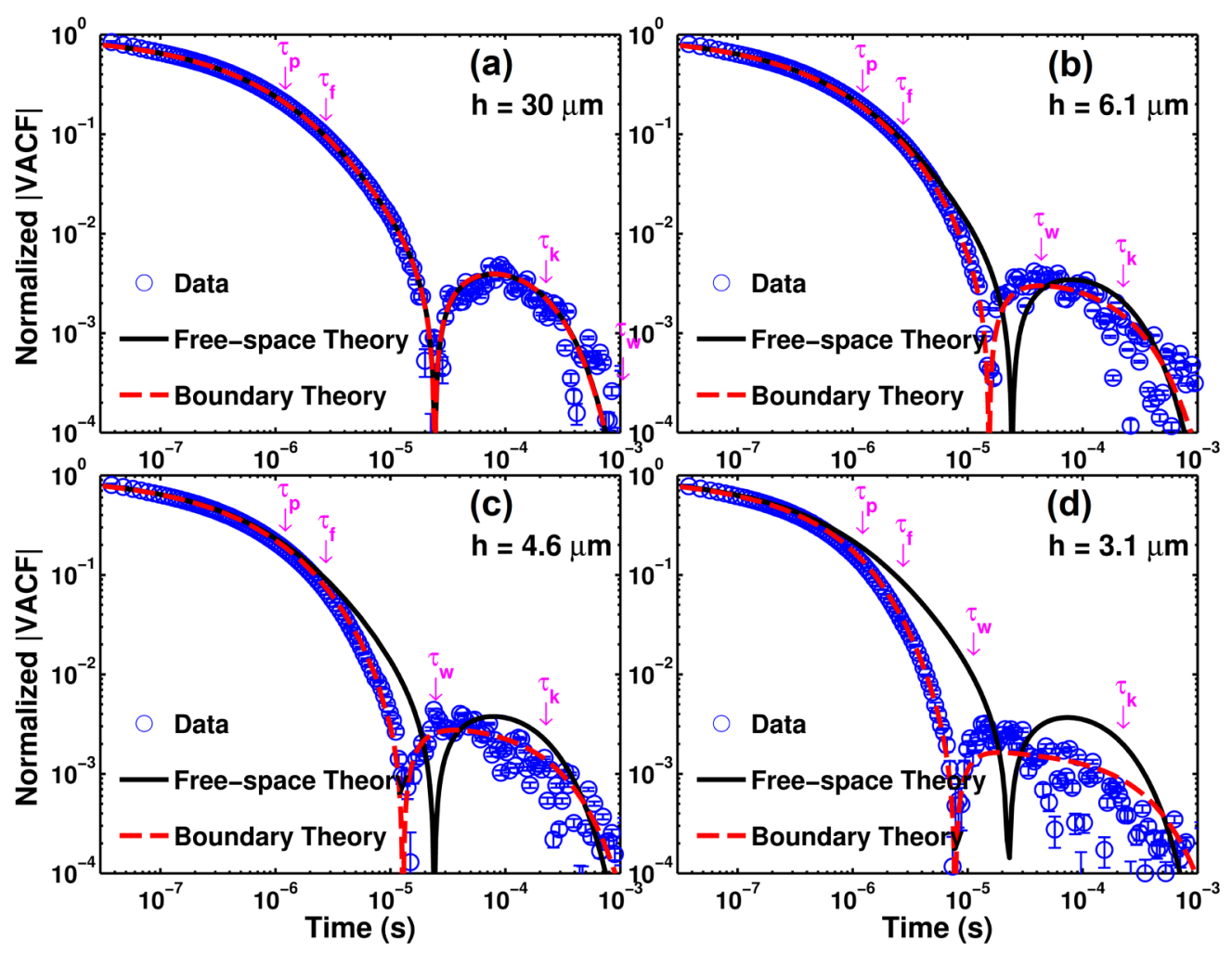

FIG. 4. (Color online) Experimental and theoretical velocity autocorrelation functions (VACFs) at the same positions as in Fig. 3 in the perpendicular direction with respect to the wall. The plots show the absolute value (normalized to $\left.\left\langle\left(v_{\perp}^{*}\right)^{2}\right\rangle=k_{B} T / m_{\perp}^{*}\right)$ on a $\log -\log$ scale. The sharp cusplike features correspond to zero crossings. (a) With a sphere-wall separation ( $h$ ) of $30 \mu \mathrm{m}\left(\tau_{w}=0.9 \mathrm{~ms}\right)$. (b) $h=6.1 \mu \mathrm{m}\left(\tau_{w}=37 \mu \mathrm{s}\right)$. (c) $h=4.6 \mu \mathrm{m}\left(\tau_{w}=21 \mu \mathrm{s}\right)$. (d) $h=3.1 \mu \mathrm{m}\left(\tau_{w}=9.6 \mu \mathrm{s}\right)$. The blue circles are the experimental data $\left(\tau_{p}=1 \mu \mathrm{s}, \tau_{f}=2.3 \mu \mathrm{s}, \tau_{k}=190 \mu \mathrm{s}\right)$; the black lines are the unbounded theoretical predictions [33] and the red dashed lines correspond to the bounded theoretical predictions at various sphere-wall separations. The VACF decays faster as the sphere approaches the wall.

predictions to calibrate the piezo readings. It turns out that the fitting of the VACF gives the most reliable results; hence our choice. The boundary effects with sphere-wall separations larger than $10 \mu \mathrm{m}$ become so weak that the VACF fitting becomes unreliable. But for smaller separations, the VACF is evidently very sensitive to the sphere-wall separation, and therefore can be used to precisely measure this separation. The offset between the piezo reading and the actual distance is then determined by fitting a line with a slope of unity to the distance obtained from the VACF fit against the piezo readings at 10 positions. The results are shown in Fig. 2(a). With this method of calibration, the sphere-wall separations at the four positions shown in Figs. 3-7 are $30 \mu \mathrm{m}, 6.1 \mu \mathrm{m}, 4.6 \mu \mathrm{m}$, and $3.1 \mu \mathrm{m}$, respectively. This is in agreement with the piezo strain gauge readings for the latter three positions, as they differ by $1.5 \mu \mathrm{m}$.

The absolute sphere-wall separations can also be obtained by another method, namely by the measurements of the hindered diffusion coefficients. The variation of the diffusion coefficient near a no-slip wall has been well studied by numerous experiments [11-13]. Keeping the same microsphere in the trap, we reduce the optical trap strength (by reducing the power from $400 \mathrm{~mW}$ to $50 \mathrm{~mW}$ ) so as to permit the MSD to enter the diffusive regime before flattening out. We redirect the detection beam to a DC-coupled balanced detector, and acquire data with the same digitizer at a lower sampling rate of $5 \mathrm{MSa} / \mathrm{s}$. Each continuous trajectory contains $2^{25}$ samples, corresponding to about $7 \mathrm{~s}$. We take 10 such measurements at each position. The hindered diffusion coefficients are then obtained at many positions by analyzing the PACF as described below.

The diffusion of a sphere near a wall becomes anisotropic and can be separated into parallel (axial) and perpendicular (radial) directions. The hindered diffusion coefficients are a function of size of the sphere $a$ and sphere-wall separation $h$, and can be obtained by measuring the Brownian motion in the diffusive regime [39]. The translational motion of a spherical particle in a fluid trapped by an optical tweezer at low frequencies can be described using

$$
K \mathbf{x}(t)+\gamma(h) \dot{\mathbf{x}}(t)=\mathbf{F}_{\mathrm{th}}(t),
$$

where $\mathbf{F}_{\text {th }}$ is the thermal stochastic force. The inertial term is neglected as it is not significant in the long time scale motion of the particle. At long time scales $t \gg \tau_{f}$, the damping factor is approximately frequency independent but depends on the sphere-wall separation. In this regime, the equation may be easily solved to obtain the position autocorrelation function (PACF) as

$$
\langle x(\tau) x(t+\tau)\rangle=\left\langle x^{2}\right\rangle e^{-[K / \gamma(h)] t}=\left\langle x^{2}\right\rangle e^{-t / \tau_{k}},
$$

where $\tau_{k}=\gamma(h) / K$ gives the time scale during which the particle experiences a drift back toward to the trap center. With trap stiffness fixed, the larger the damping factor $\gamma(h)$, the longer it takes to drift back to the center of the trap. The 


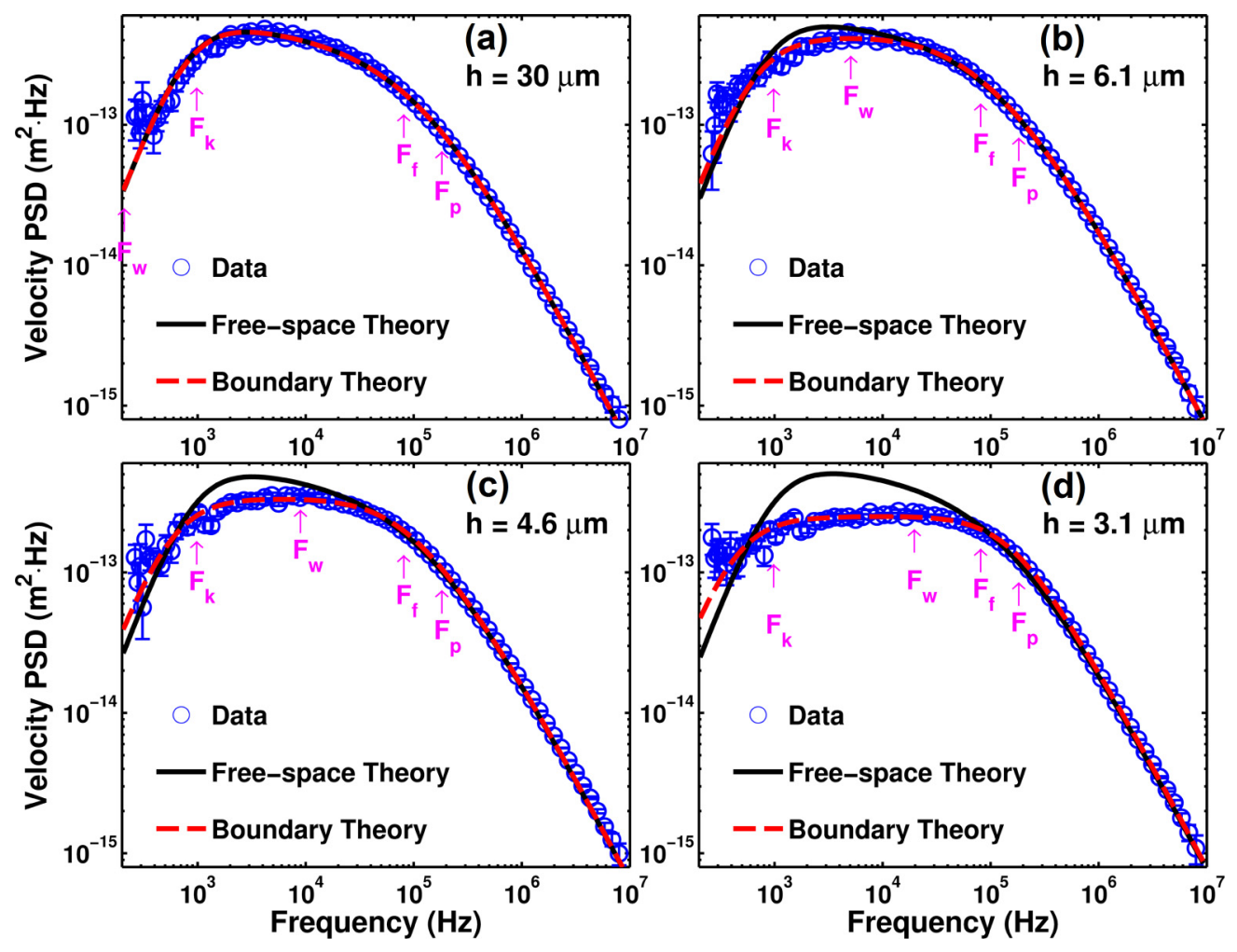

FIG. 5. (Color online) Experimental and theoretical velocity power spectrum (VPSD) at the same positions as in Fig. 3 in perpendicular direction to the wall. (a) $h=30 \mu \mathrm{m}\left(F_{w}=177 \mathrm{~Hz}\right)$. (b) $h=6.1 \mu \mathrm{m}\left(F_{w}=4.3 \mathrm{kHz}\right)$. (c) $h=4.6 \mu \mathrm{m}\left(F_{w}=7.5 \mathrm{kHz}\right)$. (d) $h=3.1 \mu \mathrm{m}$ $\left(F_{w}=16.6 \mathrm{kHz}\right)$. The blue circles are the experimental data $\left(F_{p}=153 \mathrm{kHz}, F_{f}=68 \mathrm{kHz}, F_{k}=833 \mathrm{~Hz}\right)$; the black lines are the unbounded theoretical predictions and the red dashed lines correspond to the bounded theoretical predictions at various sphere-wall separations.

diffusion coefficient $D$ can be obtained from the damping factor using the Stokes-Einstein relation $D(h)=k_{B} T / \gamma(h)$.

We are able to measure the hindered diffusion coefficients in the radial direction $D_{\widehat{r}}(h)$ with the vertical fiber as the boundary and the axial direction $D_{\bar{z}}(h)$ with the horizontal fiber as the boundary.

These measurements may then be fit to predictions obtained either from the flat-wall theory [Eq. (4) of Ref. [39] and Eq. (4) of Ref. [12]] or from the cylindrical-wall theory [40]. The cylindrical-wall theory is improved by adding a geometric series summation for higher order reflections, as suggested in Chap. 7 of Ref. [41], to obtain $D_{\widehat{r}}=D_{0}\left(1-\left(a / R_{0}\right) k_{r}\right)$, where $R_{0}$ is the radius of the cylinder, $k_{r}$ is the correction defined in Eq. (4.5) of Ref. [40], and $D_{0}$ is the free-space value of the diffusion coefficient. Similarly, the hindered diffusion coefficients in the axial and azimuthal directions can be obtained through $D_{\bar{z}}=D_{0}\left(1-\left(a / R_{0}\right) k_{z}\right)$ and $D_{\widehat{\theta}}=$ $D_{0}\left(1-\left(a / R_{0}\right) k_{\theta}\right)$, respectively (see also erratum [42]).

The diffusion coefficient in the perpendicular direction (normalized to its bulk value $D_{0}=0.14 \mu \mathrm{m}^{2} / \mathrm{s}$ ) of a $3 \mu \mathrm{m}$ sphere is shown as a function of sphere-wall separation from $2.5 \mu \mathrm{m}$ to $30 \mu \mathrm{m}$ [Fig. 2(b)]. The mobility of the sphere decreases as it approaches the wall. The absolute position offset is obtained by a least-squares fit of the measured diffusion coefficients to the flat-wall theory [41], which is the red solid line shown in Fig. 2(b). The blue dashed line is the cylindrical-wall theory for $D_{\widehat{r}}$ [40], improved as described before. The green squares with error bars are the experimental data. With this method of calibration, the sphere-wall separations at the four positions shown in Figs. 3-7 are $29.7 \mu \mathrm{m}, 5.8 \mu \mathrm{m}, 4.3 \mu \mathrm{m}$, and $2.8 \mu \mathrm{m}$, respectively. The discrepancy of the absolute distance calibration between the two methods is within $300 \mathrm{~nm}$.

The absolute positions presented in the results section were obtained by fitting the VACF. The experimental results agree with the theoretical predictions within the uncertainty irrespective of the calibration method used.

Both absolute distance calibration methods suggest that once the sphere-wall separation is larger than about $7 \mu \mathrm{m}$, the curvature of the cylindrical fiber becomes important.

\section{RESULTS}

The MSDs at four different positions (with sphere-wall separations of $30 \mu \mathrm{m}, 6.1 \mu \mathrm{m}, 4.6 \mu \mathrm{m}$, and $3.1 \mu \mathrm{m}$ ) in the perpendicular direction to the wall are shown in Fig. 3. The optical trapping causes the MSD to plateau around $\tau_{k}$, the time scale during which the particle experiences a drift back towards the trap center, before the purely diffusive regime is reached. The Brownian motion reaches the ballistic regime at time scales shorter than both $\tau_{p}$ and $\tau_{f}$ (see Sec. II B).

With a large sphere-wall separation of $h=30 \mu \mathrm{m}$, the boundary effects are negligible: as shown in Fig. 3(a), the experimental data agrees well with both free-space theory and boundary theory. Figures 3(b)-3(d) show the surface confinement effects appear on the time scale of $\tau_{w}$ (see Sec. II B). The MSD is suppressed as the sphere-wall separation decreases. When $\tau_{w}$ is comparable to $\tau_{p}$ and $\tau_{f}$, the surface confinement 


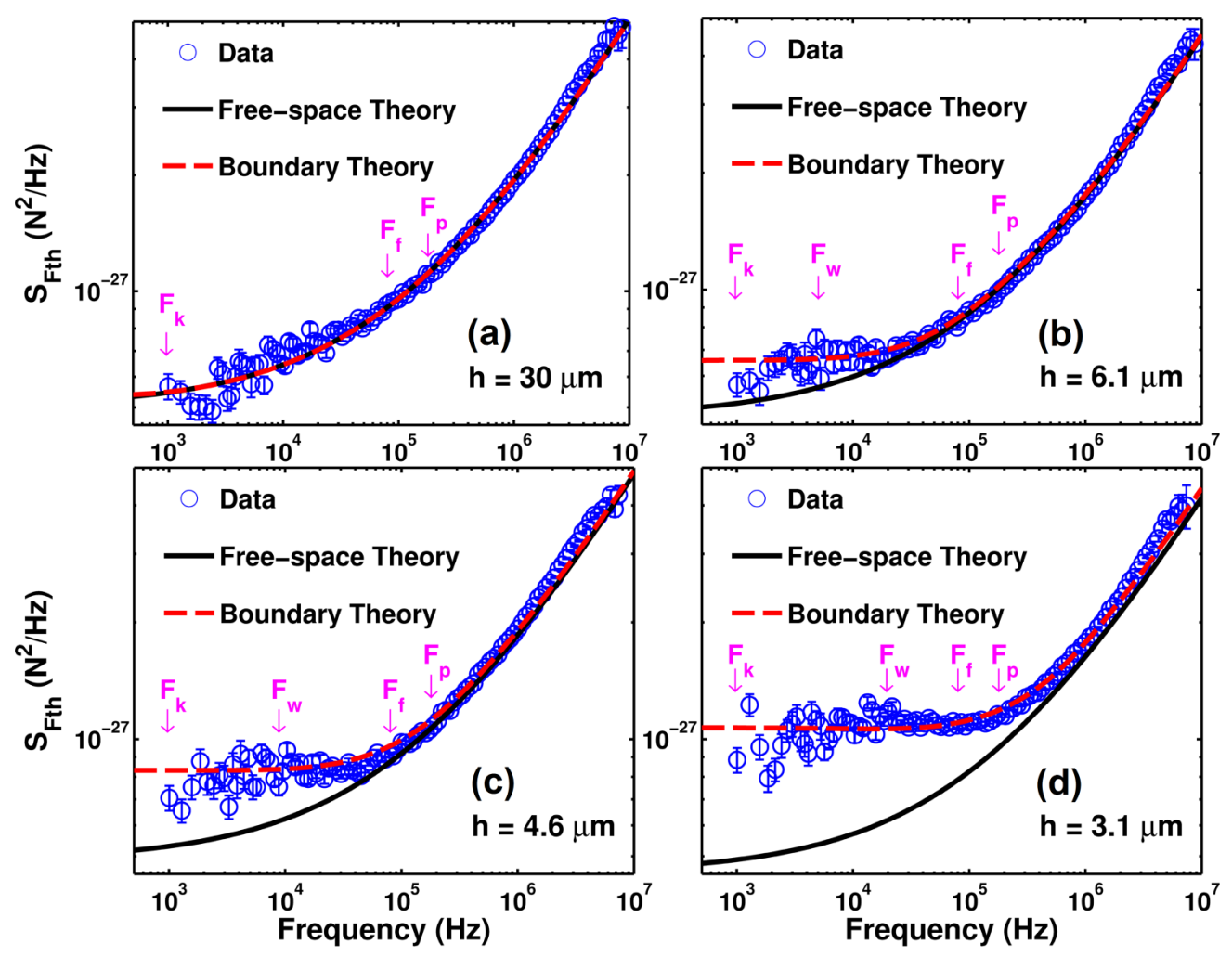

FIG. 6. (Color online) Experimental and theoretical thermal force power spectral density (FPSD) at the same positions as in Fig. 3 in perpendicular direction to the wall. (a) $h=30 \mu \mathrm{m}\left(F_{w}=177 \mathrm{~Hz}\right)$. (b) $h=6.1 \mu \mathrm{m}\left(F_{w}=4.3 \mathrm{kHz}\right)$. (c) $h=4.6 \mu \mathrm{m}\left(F_{w}=7.5 \mathrm{kHz}\right)$. (d) $h=3.1 \mu \mathrm{m}\left(F_{w}=16.6 \mathrm{kHz}\right)$. The blue circles are the experimental data $\left(F_{p}=153 \mathrm{kHz}, F_{f}=68 \mathrm{kHz}, F_{k}=833 \mathrm{~Hz}\right)$; the black lines are the unbounded theoretical predictions $[18,21]$ and the red dashed lines correspond to the bounded theoretical predictions at various sphere-wall separations.

on the motion of the particle due to boundaries not only occurs in the diffusive regime, but also in the ballistic regime.

The VACFs, normalized to $\left\langle\left(v^{*}\right)^{2}\right\rangle$ as given by Eq. (13), in the perpendicular direction to the wall, are shown in Fig. 4, for the same positions as in Fig. 3 (with $h=30 \mu \mathrm{m}, 6.1 \mu \mathrm{m}$, $4.6 \mu \mathrm{m}$, and $3.1 \mu \mathrm{m}$ ). The boundary effects are negligible with a large sphere-wall separation $h=30 \mu \mathrm{m}$, as shown in Fig. 4(a). Figures 4(b)-4(d) show that the VACF of a particle near a boundary decreases faster as the sphere-wall separation decreases. The rapid decay of the VACF reflects the loss of fluid momentum at the no-slip boundary, which is consistent with previous numerical simulations [43-45] as well as those presented in Sec. VI. The time at which the VACF near a wall starts falling remarkably below that in an unbounded fluid is related to the time scale $\tau_{w}$.

The velocity power spectral density (VPSD) is the Fourier transform of the VACF, and characterizes the distribution of power in the fluctuations of the velocity at various frequencies. The VPSD for motion perpendicular to the wall is shown in Fig. 5 with the same sphere-wall separations as in Fig. 3 (with $h=30 \mu \mathrm{m}, 6.1 \mu \mathrm{m}, 4.6 \mu \mathrm{m}$, and 3.1 $\mu \mathrm{m}$ ). The reduced correlation seen in the VACF close to the wall is seen as an increased flatness of the velocity PSD at similar time scales (or corresponding frequency scales). The frequency at which the VPSD near a wall starts to deviate remarkably from that in an unbounded fluid is related to the frequency scale $F_{w}=1 /\left(2 \pi \tau_{w}\right)$. The suppression of the velocity PSD from the free-space theory seen at these scales is complemented by an increase in the VPSD at other frequencies, thus keeping the area under the curve to $\left\langle\left(v^{*}\right)^{2}\right\rangle$.

As described in Sec. IIC, the thermal stochastic force acting on a Brownian particle is known to be "white" in the Einstein-Ornstein-Uhlenbeck model. It is also well known that the thermal force develops a colored spectrum due to hydrodynamic interactions, as has recently been experimentally verified [18,21]. Surprisingly, the thermal force loses its color at low frequencies in the presence of a boundary. The thermal force power spectral density (FPSD) acting on the particle near a no-slip wall becomes flat at low frequencies $\left(\omega \tau_{f} \ll 1\right.$ and $\left.\omega \tau_{w} \ll 1\right)$, as described in Sec. II C. This is believed to be a result of destructive interference between the incident and reflected flows, though more study is needed.

The FPSDs in the direction perpendicular to the wall are shown in Fig. 6, for the same positions as in Fig. 3 (with $h=30 \mu \mathrm{m}, 6.1 \mu \mathrm{m}, 4.6 \mu \mathrm{m}$, and $3.1 \mu \mathrm{m}$ ). Figure 6(a), which has a large sphere-wall separation of $h=30 \mu \mathrm{m}$, shows that the boundary effects are negligible and verifies the colored thermal force spectral density. Figures 6(b)-6(d) show that the FPSDs in the perpendicular direction flatten at low frequencies and their DC values increase to $4 k_{B} T \gamma_{s} /\left(1-\frac{9 a}{8 h}\right)$ from the bulk value of $4 k_{B} T \gamma_{s}$. The increase in the thermal force is consistent with the increase in the drag force, in accordance with the fluctuation-dissipation theorem [34]. It is worth noting that this flattening of the FPSD explains the suppression of a resonance in the position power spectrum of a particle near a wall observed in previous experiments $[17,18]$. 


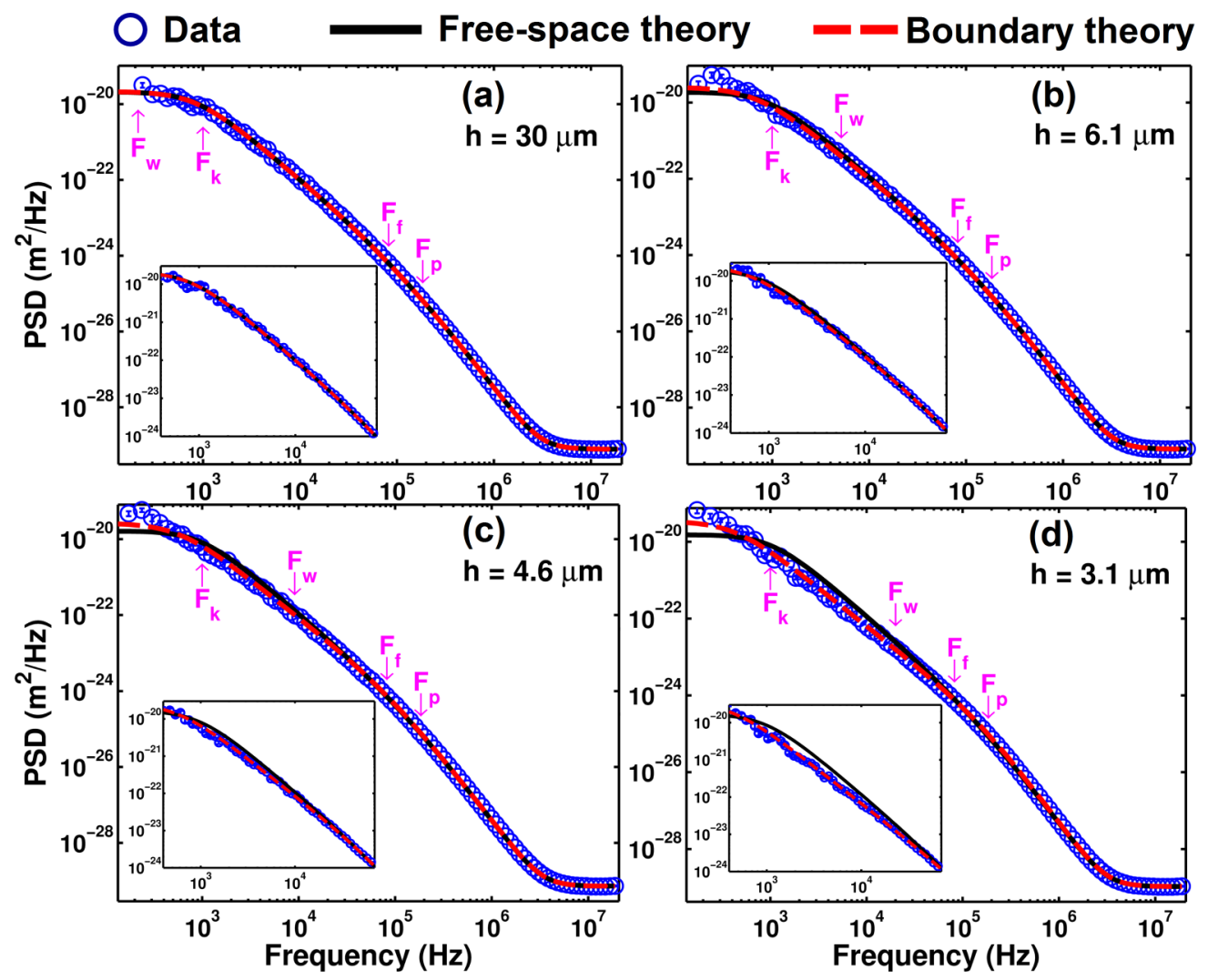

FIG. 7. (Color online) Experimental and theoretical position power spectrum (PSD) at the same positions as in Fig. 3 in perpendicular direction to the wall. (a) $h=30 \mu \mathrm{m}\left(F_{w}=177 \mathrm{~Hz}\right)$. (b) $h=6.1 \mu \mathrm{m}\left(F_{w}=4.3 \mathrm{kHz}\right)$. (c) $h=4.6 \mu \mathrm{m}\left(F_{w}=7.5 \mathrm{kHz}\right)$. (d) $h=3.1 \mu \mathrm{m}$ $\left(F_{w}=16.6 \mathrm{kHz}\right)$. The blue circles are the experimental data $\left(F_{p}=153 \mathrm{kHz}, F_{f}=68 \mathrm{kHz}, F_{k}=833 \mathrm{~Hz}\right)$; the black lines are the sum of the unbounded theoretical predictions and a constant shot noise and the red dashed lines correspond to the sum of the bounded theoretical predictions at various sphere-wall separations and a constant shot noise. At high frequencies, the position PSDs flatten at $3 \times 10^{-15} \mathrm{~m} / \sqrt{\mathrm{Hz}}$, limited by the shot noise of the detection beam [21,22].

Previous work $[17,18]$ has discussed the behavior of the position PSD at low frequencies, up to about the trap corner frequency. They observe a resonance caused by the color of the thermal force, and its suppression as the distance to the wall decreases. Our observations suggest that this suppression is due to the enhanced flatness in the FPSD near a no-slip boundary.

We complement these works by observing the position PSD and velocity PSD at frequencies higher than the trapping frequency. The position PSD for motion perpendicular to the wall is shown in Fig. 7 at four different sphere-wall distances. The DC value of the position PSD increases as we go closer to the wall (an effect that is not evident in previous work due to normalization), resulting from an increase in the thermal force at low frequencies, consistent with the increased Stokes drag coefficient. Observation of this in the experiment is obscured by low-frequency noise. However, corresponding to the increased flatness of the thermal force near the wall, the position PSD decays faster than it would in free space. We observe this effect, especially when the particle is close to the boundary. The observation is plagued by low-frequency noise at larger sphere-wall separations. At high frequencies, the position PSDs flatten at around $3 \times$ $10^{-15} \mathrm{~m} / \sqrt{\mathrm{Hz}}$, limited by the shot noise of the detection beam [21,22].
All of the observed statistical properties, namely MSD, VACF, FPSD, VPSD, and the position PSD, in the presence of the wall approach the corresponding free-space theories for time scales much shorter than $\tau_{w}$ (or frequency scales much larger than $F_{w}$ ). This is expected, as the numerical simulations (see Sec. VI) suggest that the vorticity from the wall takes time on the order of $\tau_{w}$ to reach the sphere. The inertial effects due to the boundary, which persist at high frequencies in the form of an increased effective mass, are too insignificant to discern.

The presence of a no-slip boundary has similar effects on the dynamics of the particle in the direction parallel to the boundary, except less pronounced. Thus the motion of the sphere becomes anisotropic. For brevity, we show boundary effects in the parallel direction only for a sphere-wall separation of $2.9 \mu \mathrm{m}$. We acquire and analyze the data in the parallel direction in the same way as in the perpendicular direction. The MSD, VPSD, VACF, and FPSD in the parallel direction are shown in Fig. 8.

\section{NUMERICAL SIMULATION}

We study the fluid dynamics around a sphere both in free space and near a no-slip wall numerically using COMSOL Multiphysics (COMSOL Inc., Palo Alto, CA) with a 2D axisymmetric configuration $[5,46]$. The fluid in this simulation 

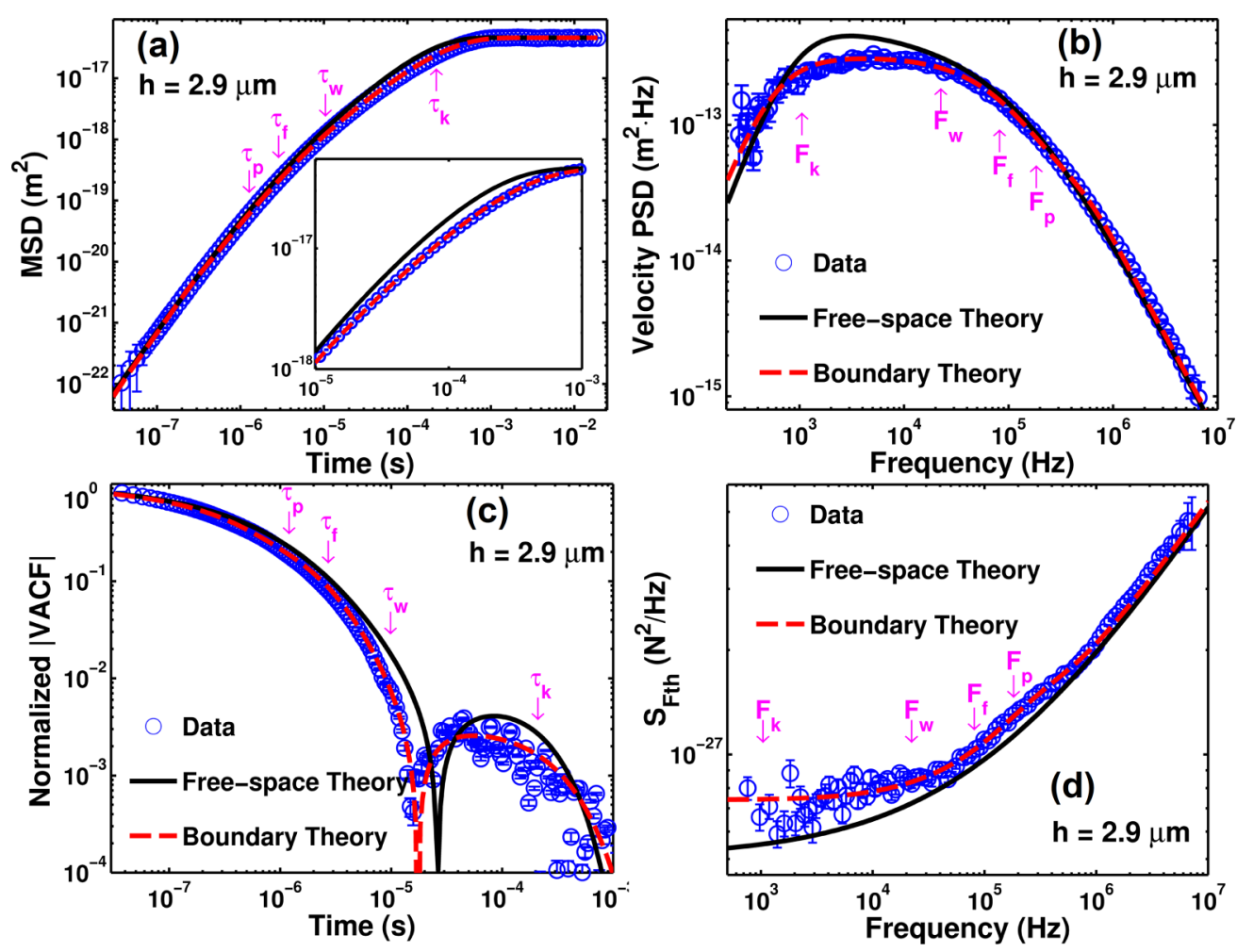

FIG. 8. (Color online) Experimental and theoretical Brownian dynamics in the parallel direction with a sphere-wall separation of $2.9 \mu \mathrm{m}$. (a) MSD. (b) VPSD. (c) Absolute VACF (normalized to $\left\langle\left(v_{\|}^{*}\right)^{2}\right\rangle=k_{B} T / m_{\|}^{*}$ ). (d) FPSD. The blue circles are the experimental data; the black lines are the unbounded theoretical predictions and the red dashed lines correspond to the bounded theoretical predictions.

has the same properties as water and the diameter of the sphere is $3 \mu \mathrm{m}$. Figure 9 shows the longitudinal section of Stokes flow field around a moving sphere both in an unbounded and bounded fluid. The white half circle represents the sphere, which is moving downwards at $1 \mu \mathrm{m} / \mathrm{s}$. The pressure field, shear stress magnitude field, velocity magnitude field, and vorticity field (azimuthal component) are plotted in a $20 \mu \mathrm{m}$ by $20 \mu \mathrm{m}$ view. The left edge represents the axis of cylindrical symmetry. The fields around the free-space sphere have up-down symmetry (antisymmetry in the pressure field), while the wall (the purple solid line) breaks this symmetry. In the bounded case, the sphere-wall separation $h$ is $3 \mu \mathrm{m}$. The fields above the sphere both in the bounded case and unbounded case are similar. The fields below the sphere are significantly altered by the wall. The fluid builds up much higher pressure and shear stress, which is responsible for the faster-decay VACF of a bounded sphere. The same conclusion has been drawn for a sphere in a confined fluid between two

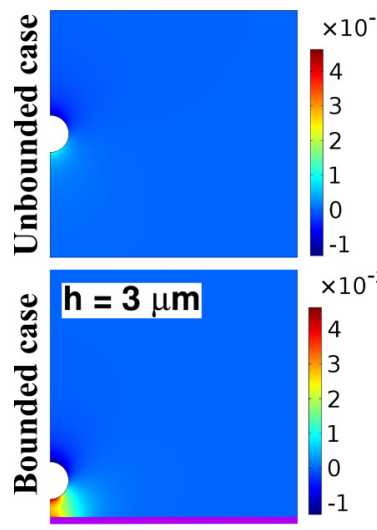

(a) Pressure field

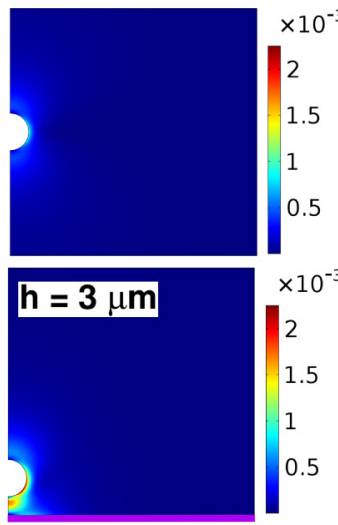

(b) Shear stress field

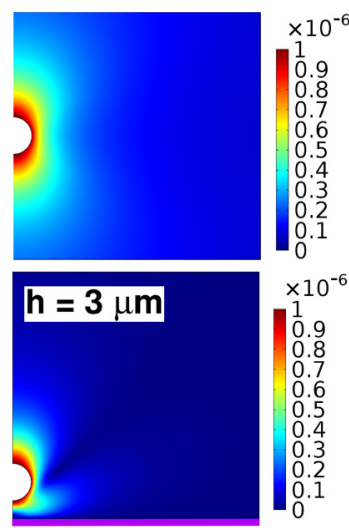

(c) Velocity field

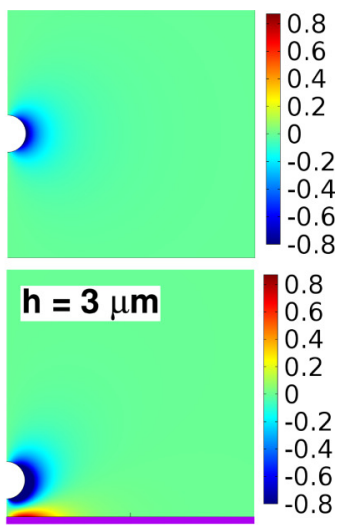

(d) Vorticity field

FIG. 9. (Color online) Fluid fields of steady Stokes flow near a sphere both in free space and bounded fluid (a) pressure field. (b) Shear stress magnitude field. (c) Velocity magnitude field. (d) Vorticity field (azimuthal component). The top four figures are for the unbounded case and the bottom four figures are for the bounded case. The white half circle represents the sphere ( $3 \mu \mathrm{m}$ diameter) and the left boundary corresponds to the axis of cylindrical symmetry. The purple solid lines represent the no-slip wall and all other boundaries are open boundaries. The sphere is moving downwards at $1 \mu \mathrm{m} / \mathrm{s}$. In the bounded case, the sphere is at position with a sphere-wall separation of $3 \mu \mathrm{m}$. 


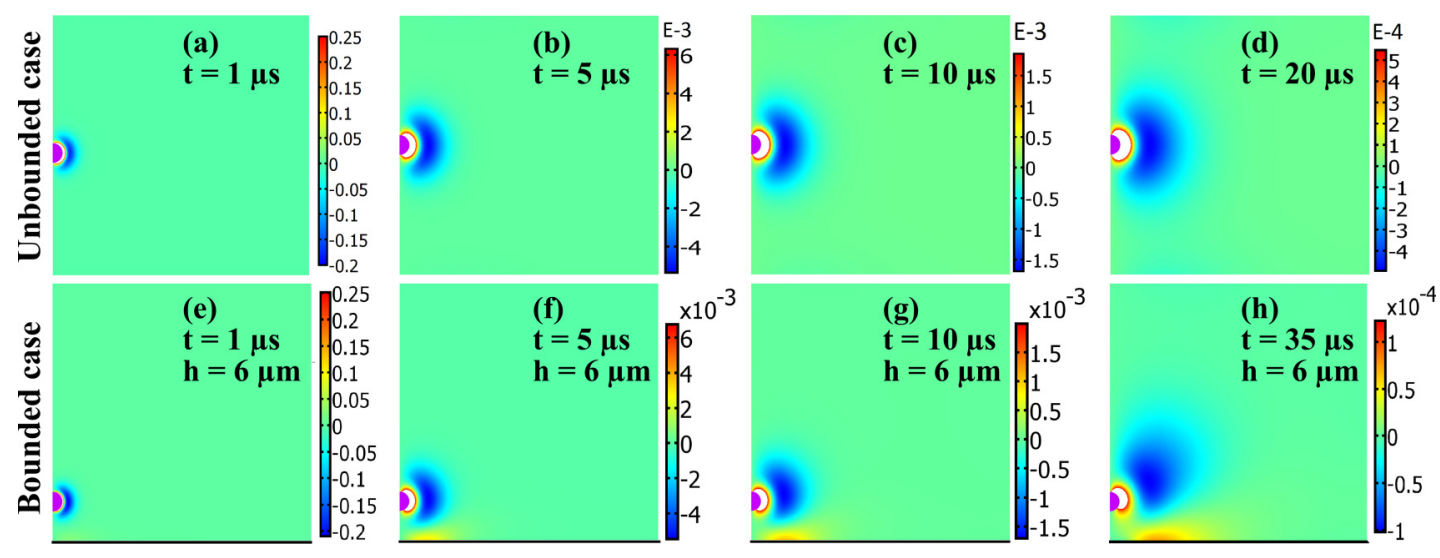

FIG. 10. (Color online) Study of the onset of boundary effects by observing the vorticity field (azimuthal component). The sphere (purple half circle, $3 \mu \mathrm{m}$ diameter), initially at rest, receives a 1- $\mu$ s impulse in the downward direction, with an acceleration of $2 \mathrm{~m} / \mathrm{s}^{2}$ in the first $0.5 \mu \mathrm{s}$ and a deceleration of $2 \mathrm{~m} / \mathrm{s}^{2}$ in the second $0.5 \mu \mathrm{s}$. Unbounded case (a) vorticity field at $\mathrm{t}=1 \mu \mathrm{s}$. (b) Vorticity field at $\mathrm{t}=5 \mu \mathrm{s}$. (c) Vorticity field at $\mathrm{t}=10 \mu \mathrm{s}$. (d) Vorticity field at $\mathrm{t}=20 \mu \mathrm{s}$. Bounded case with the sphere initially at a sphere-wall separation of $6 \mu \mathrm{m}$. (e) Vorticity field at $\mathrm{t}=1 \mu \mathrm{s}$. (f) Vorticity field at $\mathrm{t}=5 \mu \mathrm{s}$. (g) Vorticity field at $\mathrm{t}=10 \mu \mathrm{s}$. (h) Vorticity field at $\mathrm{t}=35 \mu \mathrm{s}$. The left edge is the axis of cylindrical symmetry, and top and right boundaries are open boundaries. The white areas are out-of-range clippings: the white areas should be redder than their surrounding color. The bottom edge is an open boundary in the unbounded case and a no-slip boundary in the bounded case.

flat walls by numerical simulation [43]. The fluid flow has a significant transverse component parallel to the wall, due to the presence of the impermeable boundary. Vorticity is generated not only on the surface of the sphere but also on the surface of the wall but with opposite sign.

Conventionally, the boundary effects are assumed to start becoming important around $\tau_{w}$, which is the time scale over which the vorticity generated on the surface of the sphere (or the wall) propagates across the sphere-wall separation. It turns out that $\tau_{w}$ is just a rough estimation. Our data (both in VACF and MSD) starts to deviate from the free-space theory well before $\tau_{w}$. We study the onset of boundary effects by observing the dynamics of the vorticity field (azimuthal component), as shown in Fig. 10. Initially, both the $3 \mu \mathrm{m}$ diameter sphere and fluid are at rest. At $t=0$ the sphere receives a $1 \mu \mathrm{s}$ downwards impulse accelerating with a constant acceleration of $2 \mathrm{~m} / \mathrm{s}^{2}$ for $0.5 \mu \mathrm{s}$ and then decelerates with a constant deceleration of $2 \mathrm{~m} / \mathrm{s}^{2}$ to a stop in another $0.5 \mu \mathrm{s}$. The vorticity field (region shown is $40 \mu \mathrm{m} \times 40 \mu \mathrm{m}$ ) shows up-down symmetry in the unbounded case, which is plotted in the top half [Figs. 10(a)-10(d)]. In the bounded case [Figs. 10(e)-10(h)], the sphere moves in the same way except near a no-slip wall with a sphere-wall separation of $6 \mu \mathrm{m}$. The wall starts to break the vorticity field symmetry in a visible way around $5 \mu \mathrm{s}$, which is well before $\tau_{w}=36 \mu \mathrm{s}$. It must be noted that in addition to vorticity diffusion, there are inertial effects of the boundary at much shorter time scales due to the propagation of sound waves. To the approximation that the fluid is incompressible, these effects occur almost instantaneously. However, these effects are difficult to discern in our experimental work.

\section{DISCUSSION}

A Brownian particle located near a flat wall provides a model system to study the behavior of more complex systems whose boundaries can be modeled as effective walls, such as blood vessels, cell membranes, and a variety of microfluidic geometries. Our techniques will find broad applications in precise biophysical measurements [8,9], and in particular are capable of significantly speeding up the technique of thermal noise imaging [47]. Using a micro- or nanosphere as a remote sensor to measure the distances to nearby boundaries in porous media can potentially map out boundary contours and build a 3D microscope. The theory of boundary effects on Brownian motion near full-slip boundaries predicts significant qualitative differences [36]. Future work will study the effects of partial and full slip boundaries $[36,48,49]$ on Brownian motion with these techniques.

\section{ACKNOWLEDGMENTS}

We thank Dr. Simon Kheifets, Dr. Philip Morrison, and Dr. Ubbo Felderhof for helpful discussions, David Riegler for technical help, and Dr. Sangjin Ryu and Dr. Jung Min Oh for sharing their numerical simulations. We acknowledge the support from the Sid W. Richardson Foundation and the R. A. Welch Foundation Grant No. F-1258.
[1] T. M. Squires, Rev. Mod. Phys. 77, 977 (2005).

[2] K. van Ommering, J. H. Nieuwenhuis, L. J. van IJzendoorn, B. Koopmans, and M. W. J. Prins, Appl. Phys. Lett. 89, 142511 (2006).

[3] T. M. Squires, R. J. Messinger, and S. R. Manalis, Nat. Biotechnol. 26, 417 (2008).
[4] E. Yariv and T. Miloh, J. Fluid Mech. 622, 195 (2009).

[5] S. Ryu and P. Matsudaira, Biophys. J. 98, 2574 (2010).

[6] X. L. Gong, F. Yang, S. H. Xuan, L. H. Zong, W. Zhu, and W. Q. Jiang, Phys. Rev. E 84, 061505 (2011).

[7] J. K. Huan and S. J. Jeng, J. Colloid Interface Sci. 183, 458 (1996). 
[8] E. Lauga, W. R. DiLuzio, G. M. Whitesides, and H. A. Stone, Biophys. J. 90, 400 (2006).

[9] G. Li, L.-K. Tam, and J. X. Tang, Proc. Natl. Acad. Sci. USA 105, 18355 (2008).

[10] H. Lorentz, Abhandlungen über theoretische Physik (Teubner, Leipzig, 1907), pp. 23-42.

[11] K. Ishii, T. Iwai, and H. Xia, Opt. Express 18, 7390 (2010).

[12] M. D. Carbajal-Tinoco, R. Lopez-Fernandez, and J. L. ArauzLara, Phys. Rev. Lett. 99, 138303 (2007).

[13] L. Liu, A. Woolf, A. W. Rodriguez, and F. Capasso, Proc. Natl. Acad. Sci. USA 111, E5609 (2014).

[14] C. Ha, H. Ou-Yang, and H. K. Pak, Phys. A Stat. Mech. Appl. 392, 3497 (2013).

[15] S. Jeney, B. Lukić, J. A. Kraus, T. Franosch, and L. Forró, Phys. Rev. Lett. 100, 240604 (2008).

[16] T. Franosch and S. Jeney, Phys. Rev. E 79, 031402 (2009).

[17] A. Jannasch, M. Mahamdeh, and E. Schäffer, Phys. Rev. Lett. 107, 228301 (2011).

[18] T. Franosch, M. Grimm, M. Belushkin, F. M. Mor, G. Foffi, L. Forro, and S. Jeney, Nature (London) 478, 85 (2011).

[19] T. Li, S. Kheifets, D. Medellin, and M. G. Raizen, Science 328, 1673 (2010).

[20] R. Huang, I. Chavez, K. M. Taute, B. Lukić, S. Jeney, M. G. Raizen, and E.-L. Florin, Nat. Phys. 7, 576 (2011).

[21] S. Kheifets, A. Simha, K. Melin, T. Li, and M. G. Raizen, Science 343, 1493 (2014).

[22] J. Mo, A. Simha, S. Kheifets, and M. G. Raizen, Opt. Express 23, 1888 (2015).

[23] J. N. Israelachvili, Intermolecular and Surface Forces (Elsevier Science, Burlington, 2010).

[24] M. A. Bevan and D. C. Prieve, Langmuir 15, 7925 (1999).

[25] J. N. Munday, F. Capasso, and V. A. Parsegian, Nature (London) 457, 170 (2009).

[26] B. U. Felderhof, J. Phys. Chem. B 109, 21406 (2005).

[27] A. Simha, J. Mo, and P. J. Morrison (unpublished).
[28] P. Mazur and D. Bedeaux, Physica 76, 235 (1974).

[29] B. U. Felderhof, Phys. A Stat. Mech. Appl. 84, 569 (1976).

[30] R. Zwanzig and M. Bixon, J. Fluid Mech. 69, 21 (1975).

[31] H. Lamb, Hydrodynamics (Cambridge University Press, London, 1916), pp. 181-182.

[32] B. U. Felderhof, J. Phys. Chem. B 110, 13304 (2006).

[33] H. J. H. Clercx and P. P. J. M. Schram, Phys. Rev. A 46, 1942 (1992).

[34] R. Kubo, Rep. Prog. Phys. 29, 255 (1966).

[35] G. E. Uhlenbeck and L. S. Ornstein, Phys. Rev. 36, 823 (1930).

[36] B. U. Felderhof, Phys. Rev. E 85, 046303 (2012).

[37] K. Berg-Sørensen and H. Flyvbjerg, Rev. Sci. Instrum. 75, 594 (2004).

[38] E. Tuck, Math. Comput. 21, 239 (1967).

[39] A. Pralle, E.-L. Florin, E. H. K. Stelzer, and J. K. H. Hörber, Appl. Phys. A: Mater. Sci. Process. 66, S71 (1998).

[40] M. S. Alam, K. Ishii, and H. Hasimoto, J. Phys. Soc. Jpn. 49, 405 (1980).

[41] J. Happel and H. Brenner, Low Reynolds Number Hydrodynamics With Special Applications to Particulate Media (Springer, New York, 1983).

[42] M. S. Alam, K. Ishii, and H. Hasimoto, J. Phys. Soc. Jpn. 52, 3691 (1983).

[43] R. Tatsumi and R. Yamamoto, Phys. Fluids 25, 046101 (2013).

[44] H.-Y. Yu, D. M. Eckmann, P. S. Ayyaswamy, and R. Radhakrishnan, Phys. Rev. E 91, 052303 (2015).

[45] K. Huang and I. Szlufarska, Nat. Commun. 6, 8558 (2015).

[46] H. B. Eral, J. M. Oh, D. van den Ende, F. Mugele, and M. H. G. Duits, Langmuir 26, 16722 (2010).

[47] C. Tischer, S. Altmann, S. Fišinger, J. K. H. Hörber, E. H. K. Stelzer, and E.-L. Florin, Appl. Phys. Lett. 79, 3878 (2001).

[48] G. M. Wang, R. Prabhakar, and E. M. Sevick, Phys. Rev. Lett. 103, 248303 (2009).

[49] A. Erbaş, R. Podgornik, and R. R. Netz, Eur. Phys. J. E 32, 147 (2010). 